Document downloaded from:

http://hdl.handle.net/10251/176990

This paper must be cited as:

Felipe Román, MJ.; Kazarin, LS.; Martínez-Pastor, A.; Ortiz-Sotomayor, VM. (2020). On products of groups and indices not divisible by a given prime. Monatshefte für Mathematik. 193(4):811-827. https://doi.org/10.1007/s00605-020-01446-z

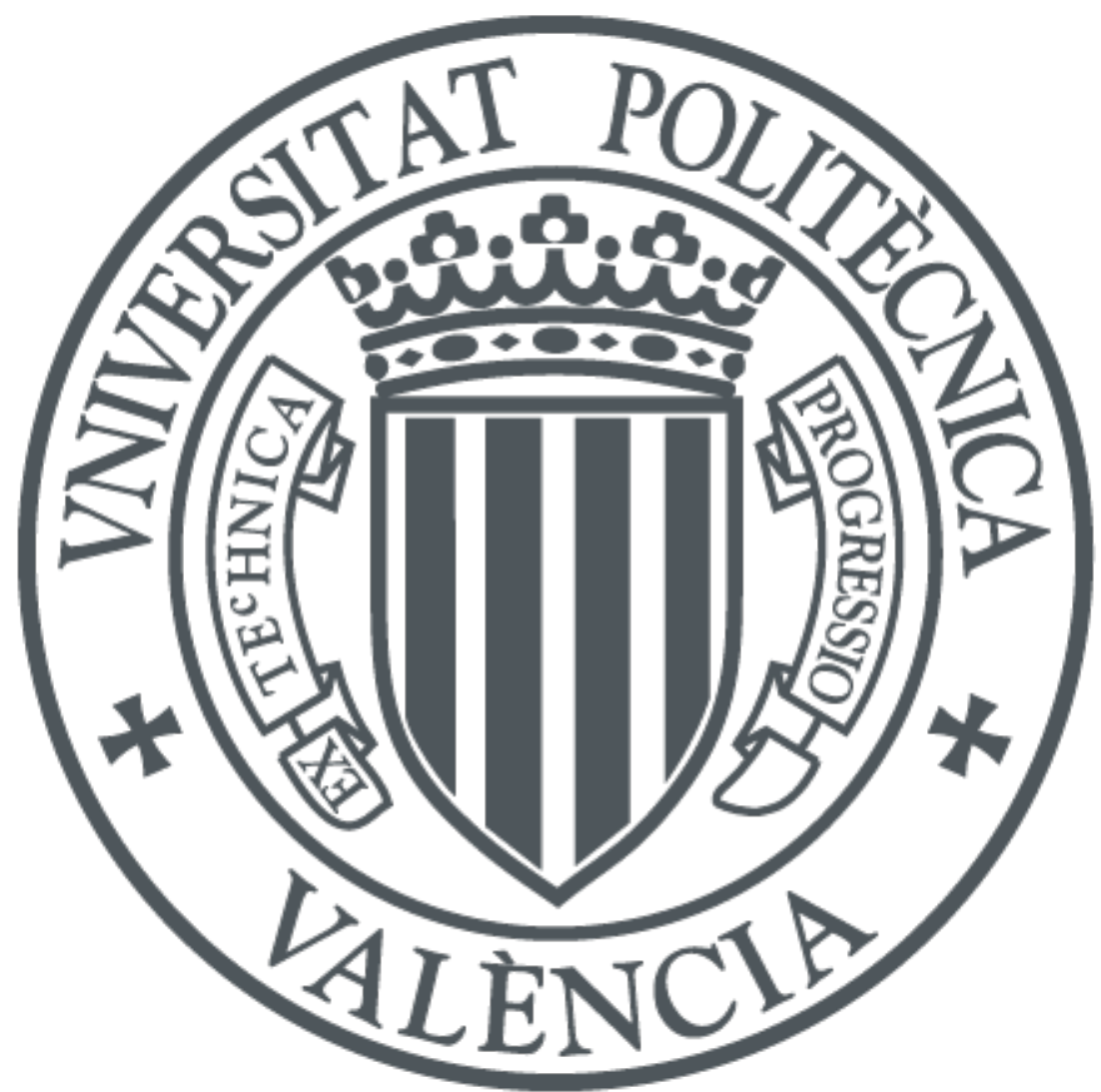

The final publication is available at

https://doi.org/10.1007/s00605-020-01446-z

Copyright Springer-Verlag

Additional Information 


\title{
On products of groups and indices not divisible by a given prime
}

\author{
María José Felipe · Lev S. Kazarin · Ana \\ Martínez-Pastor · Víctor Sotomayor \\ Dedicated to the memory of Carlo Casolo
}

\begin{abstract}
Let the group $G=A B$ be the product of subgroups $A$ and $B$, and let $p$ be a prime. We prove that $p$ does not divide the conjugacy class size (index) of each $p$ regular element of prime power order $x \in A \cup B$ if and only if $G$ is $p$-decomposable, i.e. $G=O_{p}(G) \times O_{p^{\prime}}(G)$.
\end{abstract}

Keywords Finite groups · Products of groups · Conjugacy classes $\cdot p$-structure Prime graph $\cdot$ Almost simple groups

Mathematics Subject Classification (2010) 20D40 20 E45 20 D20 20 D60

\section{Introduction and statement of results}

All groups considered in this paper are finite. In recent years a new research line its being developed in the confluence of two well-established areas of study in group theory. On the one hand the theory of products of groups, and on the other hand the study of the influence of conjugacy class sizes, also called indices, on the structure of finite groups. The present paper is a contribution to this current development.

Research supported by Proyecto PGC2018-096872-B-I00 from the Ministerio de Ciencia, Innovación y Universidades, Spain, and FEDER. The second author is also supported by Project VIP-008 of Yaroslavl P. Demidov State University and the third author by Proyecto PROMETEO/2017/057 from the Generalitat Valenciana, Spain

M.J. Felipe · A. Martínez-Pastor · V. Sotomayor

Instituto Universitario de Matemática Pura y Aplicada (IUMPA-UPV), Universitat Politècnica de València, Camino de Vera s/n, 46022, Valencia, Spain

E-mail: mfelipe@mat.upv.es, anamarti@mat.upv.es, vicorso@doctor.upv.es

ORCID ID: 0000-0002-6699-3135, 0000-0002-0208-4098, 0000-0001-8649-5742

L.S. Kazarin

Department of Mathematics, Yaroslavl P. Demidov State University, Sovetskaya Str 14, 150014 Yaroslavl, Russia

E-mail: Kazarin@uniyar.ac.ru

ORCID ID: 0000-0003-1836-0955 
Regarding products of groups, the main objective is to obtain information about the structure of a factorised group from the one of the subgroups in the factorization (and vice versa). In this setting, the fact that a product of two normal supersoluble groups is not necessarily supersoluble, has led to the approach of assuming certain permutability relations between the factors involved (see [3] for a detailed account). In particular, among others, mutually permutable products have been considered. These are factorised groups $G=A B$ such that $A$ permutes with every subgroup of $B$ and $B$ permutes with every subgroup of $A$.

Besides, during the last decades, several authors have carried out in-depth investigations with the purpose of understanding how the structure of a finite group is affected by the indices of its elements. In particular, it has been examined whether the indices of some subsets of elements are enough in order to provide features of the group. The survey [4] gives a general overview about this subject until 2011.

In the recent approach, which combines the above mentioned lines within the theory of groups, the main aim is to analyze how the indices of some elements in the factors of a factorised group influence the structure of the whole group. Most of the contributions in this framework consider additional hypotheses on the subgroups in the factorization. Some of them $([19,25])$ impose some (sub)normality conditions on either both factors. Other papers consider mutually permutable products (see $[2,7,11]$ ). Recent work done by some of the authors $([13,14])$ extends previous developements by considering some special type of factorisations, the so-called core-factorisations. Only [12] treats prime power indices without considering any additional restriction on the factors. On the other hand, it is to be said that in most cases the conditions on the indices are imposed only on some subsets of elements of the factors, namely prime power order elements, $p$-regular elements, zeros of irreducible characters, etc.

The notation and terminology are as follows. For an element $x$ in a group $G$, we call $i_{G}(x)$ the index of $x$, i.e $i_{G}(x)=\left|G: \mathrm{C}_{G}(x)\right|$. A $p$-regular element is an element whose order is not divisible by $p$, where $p$ will always be a prime number. If $n$ is a positive integer, $n_{p}$ denotes the highest power of $p$ dividing $n$. We represent by $\pi(G)$


and $\operatorname{Hall}_{\pi}(G)$ is the set of all Hall $\pi$-subgroups of $G$ for a set of primes $\pi$. A group such that $G=\mathrm{O}_{\pi}(G) \times \mathrm{O}_{\pi^{\prime}}(G)$ is said to be $\pi$-decomposable. If $H$ is a subgroup of $G$, we denote by $H^{G}$ the normal closure of $H$ in $G$. The remaining notation and terminology are standard within the theory of finite groups, and they mainly follow those of the book [8], apart from some terminology on simple groups which will be introduced later.

It is well known that if $p$ does not divide $i_{G}(x)$ for every $p$-regular element in a group $G$, then the Sylow $p$-subgroup is a direct factor of $G$ (see for instance [4, Lemma 2]). This result was improved in [19, Theorem 5] by proving that the same conclusion remains true if the conditions on the indices are only imposed on $p$-regular elements of prime power order. In this paper, we deal with the corresponding result for factorised groups, but avoiding the consideration of any additional conditions on the factors, as were considered in $[2,14,25]$. This means that, in contrast to some of the mentioned results whose proofs are elementary, the classification of finite simple groups (CFSG) has been used in our proof. In particular, we derive some results on 
the center of the prime graph of an almost simple group, which will be used as a tool.

The aim of this paper is then to prove the following result:

Main Theorem Let the group $G=A B$ be the product of subgroups $A$ and $B$, and let $p$ be a prime. Then $p$ does not divide $i_{G}(x)$ for every $p$-regular element of prime power order $x \in A \cup B$ if and only if $G$ is p-decomposable, i.e. $G=O_{p}(G) \times O_{p^{\prime}}(G)$.

Note that if $G$ is $p$-decomposable, then clearly the conditions on the indices hold. For the converse, the following lemma shows that only the existence of a unique Sylow $p$-subgroup should be proved.

Lemma 1 Let the group $G=A B$ be the product of the subgroups $A$ and $B$, and let $p$ be a prime. If $p$ does not divide $i_{G}(x)$ for every p-regular element of prime power order $x \in A \cup B$, then the following statements are equivalent:

i) G is p-closed, i.e. G has a normal Sylow p-subgroup.

ii) $G$ is p-decomposable.

Proof Clearly, it is enough to prove that (i) implies (ii). Let $P \in \operatorname{Syl}_{p}(G)$ and assume that $P \unlhd G$. Since $p$ does not divide $i_{G}(x)=\left|G: \mathrm{C}_{G}(x)\right|$ it follows that $P \leq \mathrm{C}_{G}(x)$ for every $p$-regular element of prime power order $x \in A \cup B$. Since $G$ is $p$-separable, by Lemma 3, we may consider $H$ a Hall $p^{\prime}$-subgroup of $G$ such that $H=(H \cap A)(H \cap B)$. Hence, for every element $x \in(H \cap A) \cup(H \cap B)$ of prime power order, it holds that $P \leq \mathrm{C}_{G}(x)$. Therefore $[P, H]=1$ and (ii) follows.

As an inmediate consequence of the Main Theorem, we get:

Corollary 1 Let the group $G=A B$ be the product of subgroups $A$ and $B$, and let $p$ be a prime. Then $p$ does not divide $i_{G}(x)$ for every element of prime power order $x \in A \cup B$ if and only if $G$ has a central Sylow p-subgroup, i.e. $G=O_{p}(G) \times O_{p^{\prime}}(G)$ with $O_{p}(G)$ abelian.

Our results provide an improvement of [2, Theorem 1.1] in the case of only two factors, since in that paper products of $n$ pairwise mutually permutable subgroups were considered.

Corollary 2 ([2, Theorem 1.1]) Let the group $G=A B$ be the mutually product of the subgroups $A$ and $B$, and let $p$ be a prime. Then:

i) No index $i_{G}(x)$, where $x$ is a p-regular element in $A \cup B$, is divisible by $p$ if and only if $G=O_{p}(G) \times O_{p^{\prime}}(G)$.

ii) $i_{G}(x)$ is not divisible by $p$ for every element $x \in A \cup B$ if and only if $G=O_{p}(G) \times$ $O_{p^{\prime}}(G)$ with $O_{p}(G)$ abelian.

Finally, we also point out that [14, Theorem A] and [25, Theorem 3.2] when $\pi=p^{\prime}$ are direct consequences from our main result. 


\section{Preliminary results}

We will use without further reference the following elementary lemma:

Lemma 2 Let $N$ be a normal subgroup of a group $G$, and $H$ be a subgroup of $G$. We have:

(a) $i_{N}(x)$ divides $i_{G}(x)$, for each $x \in N$.

(b) $i_{G / N}(x N)$ divides $i_{G}(x)$, for each $x \in G$.

(c) If $x N$ is a $\pi$-element of $H N / N$, for a set of primes $\pi$, then there exists a $\pi$-element $x_{1} \in H$ such that $x N=x_{1} N$.

We will also need the following fact about Hall subgroups of factorised groups, which is a convenient reformulation of $[1,1.3 .2]$. We recall that a group is a $D_{\pi^{-}}$ group, for a set of primes $\pi$, if every $\pi$-subgroup is contained in a Hall $\pi$-subgroup, and any two Hall $\pi$-subgroups are conjugate. It is well known that any $\pi$-separable group is a $\mathrm{D}_{\pi}$-group. Also, all finite groups are $\mathrm{D}_{\pi}$-groups when $\pi$ consists of a single prime.

Lemma 3 Let $G=A B$ be the product of the subgroups $A$ and $B$. Asume that $A, B$,

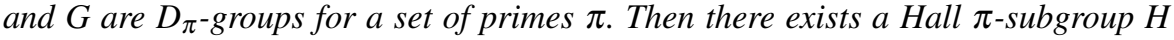
of $G$ such that $H=(H \cap A)(H \cap B)$, with $H \cap A$ a Hall $\pi$-subgroup of $A$ and $H \cap B a$ Hall $\pi$-subgroup of $B$.

Next we record some arithmetical lemmas, that will be applied later.

Lemma 4 ([16, Lemma 6]) Let $G$ be the symmetric group of degree $k$ and let $s$ be a prime. If $s^{N}$ is the largest power of $s$ dividing $|G|=k$ !, then $N \leq \frac{k-1}{s-1}$.

Lemma 5 ([24, Lemma 6(iii)]) Let $q, s, t$ be positive integers. Then:

(a) $\left(q^{s}-1, q^{t}-1\right)=q^{(s, t)}-1$,

(b) $\left(q^{s}+1, q^{t}+1\right)= \begin{cases}q^{(s, t)}+1 & \text { if both } s /(s, t) \text { and } t /(s, t) \text { are odd, } \\ (2, q+1) & \text { otherwise, }\end{cases}$

(c) $\left(q^{s}-1, q^{t}+1\right)= \begin{cases}q^{(s, t)}+1 & \text { if } s /(s, t) \text { is even and } t /(s, t) \text { is odd } \\ (2, q+1) & \text { otherwise. }\end{cases}$

We introduce now some additional terminology. Let $n$ be a positive integer and $p$ be a prime number. A prime $r$ is said to be primitive with respect to the pair $(p, n)$ (or a primitive prime divisor of $p^{n}-1$ ) if $r$ divides $p^{n}-1$ but $r$ does not divide $p^{k}-1$ for every integer $k$ such that $1 \leq k<n$.

Lemma 6 (Zsigmondy, [26]) Let $n$ be a positive integer and $p$ a prime. Then:

(a) If $n \geq 2$, then there exists a prime r primitive with respect to the pair $(p, n)$ unless $n=2$ and $p$ is a Mersenne prime or $(p, n)=(2,6)$.

(b) If the prime $r$ is primitive with respect to the pair $(p, n)$, then $r-1 \equiv 0(\bmod n)$. In particular, $r \geq n+1$. 
The following lemmas are used when dealing with prime power order elements. We remark that the proof of the first one uses CFSG.

Lemma 7 ([10, Theorem 1]) Let $G$ be a group acting transitively on a set $\Omega$ with $|\Omega|>1$. Then there exists a prime power order element $x \in G$ which acts fixed-pointfreely on $\Omega$.

Lemma 8 Let $H$ be a subgroup of a group $G$. If every prime power order element of G lies in $\bigcup_{g \in G} H^{g}$, then $G=H$.

Proof If $H$ is normal in $G$, then every prime power order element belongs to $H$, and since $G$ is generated by such elements, we get $G=H$. So we may assume that $H$ is not normal in $G$. Note that $G$ acts on $\Omega:=\left\{H^{g}: g \in G\right\}$ transitively. If $H<G$, then certainly $|\Omega|>1$ and, by Lemma 7, there exists a prime power order element $x \in G$ acting fixed-point-freely on $\Omega$. But the hypotheses imply that $x \in H^{z}$ for some $z \in G$, so $H^{z x}=H^{z}$ and this is a contradiction.

\section{Preliminaries on (almost) simple groups and their prime graphs}

We begin this section with a useful result on the centralisers of automorphisms of simple groups, which is a refinement of [9, Lemma 2.6]. In fact, the own proof of that lemma provides this stronger result:

Lemma 9 Let $N$ be a simple group. Then there exists $r \in \pi(N) \backslash \pi(\operatorname{Out}(N))$ such that $\left(r,\left|\mathrm{C}_{N}(\alpha)\right|\right)=1$ for every non-trivial $\alpha \in \operatorname{Out}(N)$ of order coprime to $|N|$.

Proof Following the proof of [9, Lemma 2.6], we can assume that $N=G(q)$ is a simple group of Lie type, with $q=p^{e}, p$ a prime and $e \geq 3$ a positive integer. In that proof it is shown that the prime $r$ is in fact a primitive prime divisor of $p^{e m}-1$ for some integer $m \geq 2$, and that such $r$ always exist under the given assumptions. Now having in mind the orders of the outer automorphisms of the simple groups of Lie type (see for instance [18, Table 2.1]) and applying Lemma 6 we can deduce that $r \notin \pi(\operatorname{Out}(N))$ (see also [18, 2.4. Proposition B]).

We will denote the prime graph of a group $G$, also called the Grünberg-Kegel graph, by $\Gamma(G)$. The set of vertices of such graph is the set $\pi(G)$ of prime divisors of $|G|$, and two vertices $r, s$ are adjacent in $\Gamma(G)$ if there exists an element of order $r s$ in $G$. The connected components of the prime graph of a simple group are known from [23] and [17]. We will denote by $\mathrm{Z}(\Gamma(G))$, the center of the graph, i.e. $\mathrm{Z}(\Gamma(G))=$ $\{p \mid p$ is adjacent to $r, \forall r \in \pi(G)\}$.

The following result on the center of the prime graph of alternating and symmetric groups will be used later:

Lemma 10 Let $n \geq 5, n \neq 6$ be a positive integer. Let $k$ be the largest positive integer such that $\{n, n-1, \ldots, n-k+1\}$ are consecutive composite numbers. If $k=1$, then both $\Gamma\left(A_{n}\right)$ and $\Gamma\left(\Sigma_{n}\right)$ are non-connected. For $k \geq 2$, let $t$ be the largest prime number such that $t \leq k$. Then: 
1. If $k=2$, then $\Gamma\left(A_{n}\right)$ is non-connected and $\mathrm{Z}\left(\Gamma\left(\Sigma_{n}\right)\right)=\{2\}$.

2. If $k=3$, then $\mathrm{Z}\left(\Gamma\left(A_{n}\right)\right)=\{3\}$ and $\mathrm{Z}\left(\Gamma\left(\Sigma_{n}\right)\right)=\{2,3\}$.

3. If $k \geq 4$, then $\mathrm{Z}\left(\Gamma\left(A_{n}\right)\right)=\mathrm{Z}\left(\Gamma\left(\Sigma_{n}\right)\right)=\left\{s \in \pi\left(\Sigma_{n}\right) \mid 2 \leq s \leq t\right\}$.

Proof It is well known that two odd primes $s, u$ are adjacent in $\Gamma\left(A_{n}\right)$, and so in $\Gamma\left(\Sigma_{n}\right)$, if and only if $s+u \leq n$. On the other hand, if $s$ is an odd prime, $s$ is adjacent to 2 in $\Gamma\left(A_{n}\right)$ if and only if $s+4 \leq n$, and $2, s$ are adjacent in $\Gamma\left(\Sigma_{n}\right)$ only when $s+2 \leq n$. It is then clear that if $k=1$, then both $\Gamma\left(A_{n}\right)$ and $\Gamma\left(\Sigma_{n}\right)$ are non-connected.

Consider the prime $r:=n-k$, which is the largest prime divisor of $n$ ! by the choice of $k$. Clearly, $r>\frac{n}{2}>k=n-r \geq t$. Thus $r+t \leq n$, and we deduce that $t \in \mathrm{Z}\left(\Gamma\left(\Sigma_{n}\right)\right)$.

If $k=2$, then $r=n-2$, and so $\mathrm{Z}\left(\Gamma\left(\Sigma_{n}\right)\right)=\{2\}$. Since $r+4>n$, then $\Gamma\left(A_{n}\right)$ is non-connected.

If $k=3$, then $r=n-3$. It follows that $\mathrm{Z}\left(\Gamma\left(\Sigma_{n}\right)\right)=\{2,3\}$ and $\mathrm{Z}\left(\Gamma\left(A_{n}\right)\right)=\{3\}$.

Finally, let us suppose that $k \geq 4$, so $n \geq 11$. Take a prime $s \in \pi\left(\Sigma_{n}\right)$. If $s \leq t$, then $r+s \leq r+t \leq r+k=n$, and so $s$ lies in $\mathrm{Z}\left(\Gamma\left(\Sigma_{n}\right)\right)$. Assume now $s>t$, so $s>k$. It is known that there exist two primes $\frac{n}{2}<r_{1}<r_{2} \leq n$, and we may take $r_{2}=r$. If $s \neq r$, then $s+r=s+n-k>n$. If $s=r$, then $s+r_{1}>\frac{n}{2}+\frac{n}{2}=n$. Hence, in both cases $s \notin$ $\mathrm{Z}\left(\Gamma\left(\Sigma_{n}\right)\right)$. This proves that $\mathrm{Z}\left(\Gamma\left(\Sigma_{n}\right)\right)=\left\{s \in \pi\left(\Sigma_{n}\right): 2 \leq s \leq t\right\}$. Finally, since $k \geq 4$, then $r \leq n-4$, so $r+4 \leq n$ and $2 \in \mathrm{Z}\left(\Gamma\left(A_{n}\right)\right)$. Therefore $\mathrm{Z}\left(\Gamma\left(A_{n}\right)\right)=\mathrm{Z}\left(\Gamma\left(\Sigma_{n}\right)\right)$.

For the special case of the alternating group $A_{6}$ and its group of automorphisms we can derive the following result from [20, Lemma 2]:

Lemma 11 If $A_{6}=N \unlhd G \leq \operatorname{Aut}(N)$, then $\Gamma(G)$ is non-connected, except when $G=$ $\operatorname{Aut}(N)$. In this last case $\mathrm{Z}(\Gamma(G))=\{2\}$.

Also, for sporadic groups the following result is well known (see [6] or [23, Theorem 2] and [20, Theorem 3]):

Lemma 12 If $N$ is an sporadic simple group, then $\Gamma(N)$ is non-connected. Moreover, $\Gamma(\operatorname{Aut}(N))$ is also non-connected, except when $N=M c L$ or $N=J_{2}$, and in both cases $\mathrm{Z}(\Gamma(\operatorname{Aut}(N)))=\{2\}$.

We will use the following facts on groups of Lie type in the proof of our Main Theorem. In the sequel, for $q=t^{e}, e \geq 1$, we will denote by $q_{n}$ any primitive prime divisor of $t^{e n}-1$, i.e. primitive with respect to $(t, n e)$.

Lemma 13 For $N=G(q)$ a classical simple group of Lie type of characteristic $t$ and $q=t^{e}$, there exist primes $r, s \in \pi(N) \backslash(\pi(\operatorname{Out}(N)) \cup\{t\})$ and maximal tori $T_{1}$ and $T_{2}$ of $N$, of orders divisible by $r$ and $s$, respectively, with $\left(\left|T_{1}\right|,\left|T_{2}\right|\right)=1$, as stated in Table 1. (In such table for the case $\star$, l denotes a Mersenne prime.)

Moreover, for the groups $N$ listed in Table 2, there exist a prime $s \in \pi(N) \backslash$ $(\pi(\operatorname{Out}(N)) \cup\{t\})$ and a Sylow s-subgroup of order $s$ which is self-centralising in $N$.

If $N=L_{2}(q), C_{N}(x)$ is a $t$-group for each $t$-element $x \in N$.

If $N=L_{3}(q)$, there exists a maximal torus $T$ of order $(1 / d)\left(q^{2}+q+1\right), d=$ $(3, q-1)$, such that each prime $r \in \pi(T)$ is a primitive prime divisor of $q^{3}-1$ (for $q \neq 4)$, and $(|T|, 2 t)=1$. 
If $N=U_{3}(q)$, there exists a maximal torus $T$ of order $(1 / d)\left(q^{2}-q+1\right), d=$ $(3, q+1)$, such that each prime $r \in \pi(T)$ is a primitive prime divisor of $q^{6}-1$, and $(|T|, 2 t)=1$.

\begin{tabular}{|c|c|c|c|c|c|}
\hline$N$ & $r$ & $s$ & $\left|T_{1}\right|$ & $\left|T_{2}\right|$ & Remarks \\
\hline $\begin{array}{l}L_{n}(q) \\
n \geq 4\end{array}$ & $q_{n}$ & $\begin{array}{l}q_{n-1} \\
s=7\end{array}$ & $\frac{q^{n}-1}{(n, q-1)(q-1)}$ & $\frac{q^{n-1}-1}{(n, q-1)}$ & $\begin{array}{c}(n, q) \neq(6,2) \\
(n, q) \neq(4,4),(7,2) \\
(n, q)=(4,4)\end{array}$ \\
\hline$U_{n}(q)$ & $q_{n}$ & $q_{2(n-1)}$ & $\frac{q^{n}-1}{(n, q+1)(q+1)}$ & $\frac{\left(q^{n-1}+1\right)}{(n, q+1)}$ & $\begin{array}{c}n \text { even } \\
(n, q) \neq(4,2),(6,2)\end{array}$ \\
\hline$n \geq 4$ & $q_{2 n}$ & $q_{n-1}$ & $\frac{q^{n}+1}{(n, q+1)(q+1)}$ & $\frac{q^{n-1}-1}{(n, q+1)}$ & $\begin{array}{c}n \text { odd } \\
(n, q) \neq(7,2)\end{array}$ \\
\hline $\mathrm{PSp}_{4}(q)$ & $q_{4}$ & $\begin{array}{c}q_{2} \\
s=7 \\
s \neq 2\end{array}$ & $\frac{q^{2}+1}{(2, q-1)}$ & $\frac{\left(q^{2}-1\right)}{(2, q-1)}$ & $\begin{array}{c}q \neq 8, l \quad(\star) \\
q=8 \\
q=l\end{array}$ \\
\hline$P S p_{2 n}(q)$ & $q_{2 n}$ & $q_{2(n-1)}$ & $\frac{q^{n}+1}{(2, q-1)}$ & $\frac{\left(q^{n-1}+1\right)(q-1)}{(2, q-1)}$ & $\begin{array}{c}n \text { even } \\
(n g) \neq(4,2)\end{array}$ \\
\hline$n \geq 3$ & $q_{2 n}$ & $q_{n}$ & $\frac{q^{n}+1}{(2, q-1)}$ & $\frac{\left(q^{n}-1\right)}{(2, q-1)}$ & $\begin{array}{c}n \text { odd } \\
(n, q) \neq(3,2)\end{array}$ \\
\hline $\begin{array}{c}P \Omega_{2 n}^{-}(q) \\
n \geq 4\end{array}$ & $q_{2 n}$ & $q_{2(n-1)}$ & $\frac{q^{n}+1}{\left(4, q^{n}+1\right)}$ & $\frac{\left(q^{n-1}+1\right)(q-1)}{\left(4, q^{n}+1\right)}$ & $(n, q) \neq(4,2)$ \\
\hline$P \Omega_{2 n}^{+}(q)$ & $q_{2(n-1)}$ & $q_{n-1}$ & $\frac{\left(q^{n-1}+1\right)(q+1)}{\left(4, q^{n}-1\right)}$ & $\frac{\left(q^{n-1}-1\right)(q-1)}{\left(4, q^{n}-1\right)}$ & $\begin{array}{c}n \text { even } \\
(n, q) \neq(4,2)\end{array}$ \\
\hline$n \geq 4$ & $q_{2(n-1)}$ & $q_{n}$ & $\frac{\left(q^{n-1}+1\right)(q+1)}{\left(4, q^{n}-1\right)}$ & $\frac{q^{n}-1}{\left(4, q^{n}-1\right)}$ & $n$ odd \\
\hline
\end{tabular}

Table 1 Maximal tori for classical groups

Proof We recall that a torus is an abelian $t^{\prime}$-group. The existence of the subgroups $T_{1}$ and $T_{2}$ appearing in Table 1 can be derived from the known facts about the maximal tori in these groups (see, [5, Propositions 7-10] or [21, Lemma 1.2]). The fact that the corresponding orders of the tori are coprime in each case can be deduced easily from 


\begin{tabular}{|c|c|}
\hline$N$ & $s$ \\
\hline$L_{3}(4)$ & 7 \\
\hline$L_{6}(2)$ & 31 \\
\hline$L_{7}(2)$ & 127 \\
\hline$U_{6}(2)$ & 11 \\
\hline$U_{7}(2)$ & 43 \\
\hline$P S p_{4}(4)$ & 17 \\
\hline$P S p_{6}(2)$ & 7 \\
\hline$P S p_{8}(2)$ & 17 \\
\hline$P \Omega_{8}^{-}(2)$ & 17 \\
\hline$P \Omega_{8}^{+}(2)$ & 7 \\
\hline
\end{tabular}

Table 2 Self-centralising Sylow $s$-subgroups of order $s$

Lemma 5, while the assertion regarding the primitive prime divisors is deduced from Lemma 6.

The information in Table 2 can be found either in [6], or from the orders of maximal tori for the corresponding groups.

Note that the case $\operatorname{PSp}_{4}(2) \cong \Sigma_{6}$ has already been considered in Lemma 11 .

The assertion on $L_{2}(q)$ is well known (see for instance [5, Proposition 7]).

The existence of tori of the corresponding orders in $L_{3}(q)$ and $U_{3}(q)$ can be found in [21, Lemma 1.2], and the claim on the prime divisors is easily deduced applying Lemma 5.

Lemma 14 For $N=G(q)$ an exceptional simple group of Lie type of characteristic $t$ and $q=t^{e}$, there exist primes $r, s \in \pi(N) \backslash(\pi(\operatorname{Out}(N)) \cup\{t\})$ and maximal tori $T_{1}$ and $T_{2}$ of $N$, of orders divisible by $r$ and $s$, respectively, with $\left(\left|T_{1}\right|,\left|T_{2}\right|\right)=1$, as stated in Table 3.

In the cases denoted by $(\star), r$ and $s$ denote the largest prime divisor of $\left|T_{1}\right|$ and $\left|T_{2}\right|$, respectively.

The Tits group $N=F_{4}(2)^{\prime}$ contains a Sylow 13-subgroup of order 13 which is self-centralising.

Proof The existence of the subgroups $T_{1}$ and $T_{2}$ appearing in Table 3 can be derived from the information about the maximal tori in these groups (see [21, Lemma 1.3] and [22, Lemma 2.6]).

The fact that they are coprime can be deduced from Lemma 5 having in mind that $\left|T_{i}\right|$ divides $q^{n}-1$ when we state that $q_{n} \in \pi\left(T_{i}\right), i=1,2$, (for the case ${ }^{3} D_{4}(q)$, $\left|T_{1}\right|$ divides $q^{6}-1$ ), for all groups except ${ }^{2} B_{2}(q),{ }^{2} G_{2}(q),{ }^{2} F_{4}(q)$. In the latter cases, denoted by $(\star)$, the information can be obtained from [22, Lemma 2.8].

The previous lemmas provide the following result on the center of the prime graph of a simple group of Lie type, which can also be derived from [15, Proposition 2.9].

Corollary 3 If $N$ is a simple group of Lie type, then $\mathrm{Z}(\Gamma(N))=\emptyset$.

Proof Let $t$ be the characteristic of the group of Lie type $N$. It is well known that $t \notin \mathrm{Z}(\Gamma(N))$. If $p \in \mathrm{Z}(\Gamma(N))$, then for each prime $r \neq t$, there exists an abelian $t^{\prime}$ subgroup of $N$ whose order is divisible by $p$ and $r$. Since any abelian $t^{\prime}$-subgroup is 


\begin{tabular}{|c|c|c|c|c|c|}
\hline$N$ & $r$ & $s$ & $\left|T_{1}\right|$ & $\left|T_{2}\right|$ & Remarks \\
\hline $\begin{array}{l}G_{2}(q) \\
q>2\end{array}$ & $\begin{array}{c}q_{3} \\
r=7\end{array}$ & 96 & $q^{2}+q+1$ & $q^{2}-q+1$ & $\begin{array}{l}q \neq 4 \\
q=4\end{array}$ \\
\hline$F_{4}(q)$ & $q_{8}$ & $q_{12}$ & $q^{4}+1$ & $q^{4}-q^{2}+1$ & \\
\hline$E_{6}(q)$ & $q_{9}$ & $q_{12}$ & $\frac{q^{6}+q^{3}+1}{(3, q-1)}$ & $\frac{\left(q^{4}-q^{2}+1\right)\left(q^{2}+q+1\right)}{(3, q-1)}$ & \\
\hline$E_{7}(q)$ & $q_{9}$ & $q_{14}$ & $\frac{\left(q^{6}+q^{3}+1\right)(q-1)}{(2, q-1)}$ & $\frac{q^{7}+1}{(2, q-1)}$ & \\
\hline$E_{8}(q)$ & $q_{20}$ & $q_{24}$ & $q^{8}-q^{6}+q^{4}-q^{2}+1$ & $q^{8}-q^{4}+1$ & \\
\hline${ }^{3} D_{4}(q)$ & $q_{3}$ & $q_{12}$ & $\left(q^{3}-1\right)(q+1)$ & $q^{4}-q^{2}+1$ & \\
\hline 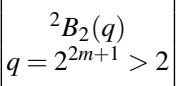 & $r$ & $s$ & $q+\sqrt{2 q}+1$ & $q-\sqrt{2 q}+1$ & $(\star)$ \\
\hline $\begin{array}{c}{ }^{2} G_{2}(q) \\
q=3^{2 m+1}>3\end{array}$ & $r$ & $s$ & $q+\sqrt{3 q}+1$ & $q-\sqrt{3 q}+1$ & $(\star)$ \\
\hline$q=2^{2 m+1}>2$ & $r$ & $s$ & $q^{2}+q \sqrt{2 q}+q+\sqrt{2 q}+1$ & $(q-\sqrt{2 q}+1)(q-1)$ & $(\star)$ \\
\hline${ }^{2} E_{6}(q)$ & $q_{18}$ & $q_{12}$ & $\frac{q^{6}-q^{3}+1}{(3, q+1)}$ & $\frac{\left(q^{4}-q^{2}+1\right)\left(q^{2}-q+1\right)}{(3, q+1)}$ & \\
\hline
\end{tabular}

Table 3 Maximal tori for exceptional groups

contained in a maximal torus, this means that $p \in \pi(T)$ for each maximal torus $T$ of $N$. Hence, the information given in Lemmas 13 and 14 leads to a contradiction.

\section{The minimal counterexample: reduction to the almost simple case}

In this section we will give a description of the structure of a minimal counterexample to our Main Theorem. Hence, having in mind Lemma 1, we assume the following hypotheses:

(H1) $p$ is a prime number.

$(\mathrm{H} 2) \mathrm{G}$ is a group satisfying the following conditions: 
(i) $G=A B$ is the product of the subgroups $A$ and $B$, and $p$ does not divide $i_{G}(x)$ for every $p$-regular element of prime power order $x \in A \cup B$.

(ii) $G$ does not have a normal Sylow $p$-subgroup.

Among all such groups we choose $(G, A, B)$ such that $|G|+|A|+|B|$ is minimal.

For such a group $G$ we have the following results.

Lemma $15 G$ has a unique minimal normal subgroup $N$ which is not a p-group. Moreover, $P \neq 1, P N \unlhd G, G / N=P N / N \times O_{p^{\prime}}(G / N)$, and $G=N N_{G}(P)$, for each $P \in \operatorname{Syl}_{p}(G)$.

Proof Since the hypotheses (H2)(i) are clearly inherited by quotients of $G$, and the class of all $p$-closed groups is a saturated formation, we deduce that $\Phi(G)=1$ and that $G$ has a unique minimal normal subgroup, say $N$. Since $G / N$ has a normal Sylow $p$-subgroup, then $\mathrm{O}_{p}(G)=1$, and so $N$ is not a $p$-group. Also this implies that $P N \unlhd G$ for each $P \in \operatorname{Syl}_{p}(G)$, and that $G / N$ is $p$-decomposable, by Lemma 1, as claimed. The last assertion follows from Frattini's argument.

From now on $N$ is the unique minimal normal subgroup of $G$.

Lemma $16 G=A P N=B P N$, for each $P \in \operatorname{Syl}_{p}(G)$.

Proof Let $P \in \operatorname{Syl}_{p}(G)$. Since $P N \unlhd G$, take for instance $T:=A P N$. Let us suppose that $T<G$. Note that $T=A(T \cap B)$. If we take any $p$-regular element of prime power order $x \in A \cup(T \cap B)$, since $G=N N_{G}(P)$, then, by our hypotheses, there exists some $n \in N \leq T$ such that $P^{n} \leq \mathrm{C}_{G}(x)$, where $P \in \operatorname{Syl}_{p}(T)$. Whence $T$ satisfies the hypotheses and, by minimality, we deduce that $P \unlhd T$. But this means, by Lemma 1 , that $T$, and so $N$, is $p$-decomposable. Since $N$ is not a $p$-group, we deduce that $N=$ $\mathrm{O}_{p^{\prime}}(N) \leq \mathrm{O}_{p^{\prime}}(T) \leq \mathrm{C}_{G}(P)$. But then $P$ is normal in $G=N N_{G}(P)$, a contradiction. Therefore, $G=A P N$ and, analogously, $G=B P N$.

Lemma 17 Either $p \in \pi(A)$ or $p \in \pi(B)$. Moreover, if $X \in\{A, B\}$ and $p \in \pi(X N)$, then $G=X N$.

Proof The first assertion is clear since $G=A B$ and $p \in \pi(G)$. Without loss of generality, let us assume that $p \in \pi(A N)$. Consider $1 \neq P_{0} \in \operatorname{Syl}_{p}(A N)$ and take $P \in \operatorname{Syl}_{p}(G)$ with $P_{0} \leq P$, that is, $P_{0}=P \cap A N$. Set $H:=A N$ and observe that $H=A(H \cap B)$. Note that for each $p$-regular element of prime power order $x \in A \cup(H \cap B)$, there exists some $n \in N$ with $P^{n} \leq \mathrm{C}_{G}(x)$. Hence $x \in \mathrm{C}_{H}\left(P^{n}\right) \leq \mathrm{C}_{H}\left(P_{0}^{n}\right)$ with $n \in N$, so $i_{H}(x)$ is not divisible by $p$. If $H<G$, then, by minimality, we deduce that $1 \neq \mathrm{O}_{p}(H)=P_{0}=$ $P \cap A N \leq A N=H$. Thus $\mathrm{O}_{p}(H)^{G}=\mathrm{O}_{p}(H)^{P}$ because $G=A P N$, by Lemma 16 . It follows that $1 \neq \mathrm{O}_{p}(H)^{G} \leq P$, and so $\mathrm{O}_{p}(G) \neq 1$, a contradiction.

Lemma 18 If $p \in \pi(N)$, then $G=A N=B N=A B$ and $N$ is a non-abelian simple group. Hence $N \unlhd G \leq \operatorname{Aut}(N)$, i.e. $G$ is an almost simple group. 
Proof The first assertion follows from Lemma 17. Since $\mathrm{O}_{p}(G)=1$ and $p \in \pi(N)$, certainly $N$ is non-abelian. Set $N=N_{1} \times N_{2} \times \cdots \times N_{r}$ with $N_{i} \cong N_{1}$ a non-abelian simple group, for $i=2, \ldots, r$, and assume that $r>1$.

Since $G=A N=B N$, both $A$ and $B$ act transitively by conjugation on the set $\Omega=\left\{N_{1}, \ldots, N_{r}\right\}$.

Suppose first that there exists some $p$-regular element of prime power order $1 \neq$ $x \in A \cup B$ such that $N_{1}^{x}=N_{i}$, for some $i>1$. By the hypotheses, there exists some $P \in \operatorname{Syl}_{p}(G)$ such that $P \leq \mathrm{C}_{G}(x)$. Moreover, $1 \neq P \cap N \in \operatorname{Syl}_{p}(N)$, and so $1 \neq$ $P \cap N_{1} \in \operatorname{Syl}_{p}\left(N_{1}\right)$. It follows that $P \cap N_{1}=\left(P \cap N_{1}\right)^{x}=P \cap N_{1}^{x}=P \cap N_{i}$, therefore $1 \neq N_{1} \cap N_{i}$, a contradiction.

Hence, we may assume that any $p$-regular element of prime power order in $A \cup B$ normalises $N_{1}$, and hence $N_{i}$ for $i=2, \ldots, r$, since $A$ and $B$ both act transitively on $\Omega$. But this means that if $R:=\cap_{i=1}^{r} N_{G}\left(N_{i}\right)$, then $G=A_{p} R=B_{p} R$, for any $A_{p} \in \operatorname{Syl}_{p}(A)$ and $B_{p} \in \operatorname{Syl}_{p}(B)$. Therefore $G=P R$ for any $P \in \operatorname{Syl}_{p}(G)$, and so $P$ acts transitively on $\Omega$. But this contradicts the fact that $1 \neq Z(P) \cap N \leq C_{N}(P)=\mathrm{C}_{N_{1}}(P) \times \cdots \times$ $\mathrm{C}_{N_{r}}(P)$, unless $r=1$. Therefore $N$ is a simple group as claimed.

In the next section the case when $N$ is a $p^{\prime}$-group will be discarded. Hence, by Lemma 18, the minimal counterexample to our Main Theorem will be an almost simple group $G, N \unlhd G \leq \operatorname{Aut}(N)$, with $p \in \pi(N)$ and $G=A B=A N=B N$.

In Section 5 we will analyse such almost simple groups satisfying the hypotheses of our Main Theorem, and all possible cases for the simple group appearing as the socle of such a group will be ruled out.

\subsection{Case $N$ is a $p^{\prime}$-group.}

Assume from now on in this section that $N$ is a $p^{\prime}$-group. Note that, in particular, $G / N$ is $p$-decomposable, by Lemma 1 , and so $G$ is $p$-separable.

Lemma $19 G=\mathrm{O}_{p^{\prime}}(G)\langle y\rangle=N \mathrm{C}_{G}(y)$, where $1 \neq y \in A$ and $\langle y\rangle \in \operatorname{Syl}_{p}(G)$. Further, $B$ is a $p^{\prime}$-group.

Proof Recall that $G / N=P N / N \times O_{p^{\prime}}(G / N)$, for $P \in \operatorname{Syl}_{p}(G)$. Now since $N$ is a $p^{\prime}$ group, it follows that $H:=\mathrm{O}_{p^{\prime}}(G) \in \operatorname{Hall}_{p^{\prime}}(G)$, so it is the unique Hall $p^{\prime}$-subgroup of $G$ and $H=(H \cap A)(H \cap B)$, by Lemma 3. We may consider $P=(P \cap A)(P \cap B)$. For some $p$-element $y \in(P \cap A) \cup(P \cap B)$, set $H_{y}:=\mathrm{O}_{p^{\prime}}(G)\langle y\rangle$. Note that $H_{y}=\left(H_{y} \cap\right.$ $A)\left(H_{y} \cap B\right)$. Now if $x \in\left(H_{y} \cap A\right) \cup\left(H_{y} \cap B\right)$ is a $p$-regular element of prime power order, then there exists some $n \in N$ with $\langle y\rangle^{n} \leq P^{n} \leq \mathrm{C}_{G}(x)$, so $x \in \mathrm{C}_{H_{y}}\left(\langle y\rangle^{n}\right)$. As $\langle y\rangle^{n}$ is a Sylow $p$-subgroup of $H_{y}$ because $n \in N \leq \mathrm{O}_{p^{\prime}}(G)$, then $H_{y}$ satisfies the hypotheses (H2). If $\left|H_{y}\right|<|G|$, by minimality we obtain that $H_{y}$ has a normal Sylow $p$-subgroup, and so $\left[y, \mathrm{O}_{p^{\prime}}(G)\right]=1$. If this holds for every $y \in(P \cap A) \cup(P \cap B)$, then $\left[P, \mathrm{O}_{p^{\prime}}(G)\right]=1$, a contradiction. Hence we may suppose that, for instance, there exists $y \in P \cap A$ with $H_{y}=\mathrm{O}_{p^{\prime}}(G)\langle y\rangle=G$. Further, since we are assuming that $|A|+|B|$ is minimal, then we deduce that $A=\left(\mathrm{O}_{p^{\prime}}(G) \cap A\right)\langle y\rangle$ and $B=\mathrm{O}_{p^{\prime}}(G) \cap B$.

Now, by coprime action and minimality, $\mathrm{O}_{p^{\prime}}(G)=\left[\mathrm{O}_{p^{\prime}}(G), y\right] \mathrm{C}_{\mathrm{O}_{p^{\prime}}(G)}(y) \leq N \mathrm{C}_{G}(y)$. Thus $G=\mathrm{O}_{p^{\prime}}(G)\langle y\rangle=N \mathrm{C}_{G}(y)$. 
Lemma $20 N \cap A \neq 1$.

Proof Assume that $N \cap A=1$. By Lemma 16, we know that $G / N=\langle y\rangle N / N \times$ $\mathrm{O}_{p^{\prime}}(G) / N$. Hence $\left[\langle y\rangle, \mathrm{O}_{p^{\prime}}(G) \cap A\right] \leq N \cap A=1$, so $\langle y\rangle$ is a Sylow $p$-subgroup of $G$ which is normal in $A$. Now, since $B$ is a $p^{\prime}$-group, we have that for any $b \in B$ of prime power order, there exists $g \in G=A B$ such that $\langle y\rangle^{g} \leq C_{G}(b)$. This implies that $\langle y\rangle^{b_{1}} \leq C_{G}(b)$ for some $b_{1} \in B$, since $\langle y\rangle^{a}=\langle y\rangle$ for any $a \in A$. It follows that each element of prime power order of $B$ lies in $\cup_{x \in B} C_{B}(\langle y\rangle)^{x}$ and so, by Lemma 8 , we deduce $[B,\langle y\rangle]=1$, a contradiction which proves our claim.

Lemma $21 N$ is a non-abelian group, so $N=N_{1} \times N_{2} \times \cdots \times N_{r}$, with $N_{i} \cong N_{1}$ a non-abelian simple group.

Proof Assume that $N$ is abelian. Therefore $\mathrm{C}_{N}(y) \leq N$ is a normal subgroup of $G=$ $N \mathrm{C}_{G}(y)$. Since $N$ is a minimal normal subgroup of $G$, we deduce that either $N=$ $\mathrm{C}_{N}(y)$ or $\mathrm{C}_{N}(y)=1$. The first case yields to the contradiction $G=\mathrm{C}_{G}(y)$. So we may assume $\mathrm{C}_{N}(y)=1$. If we take $1 \neq x \in N \cap A$ of prime power order (which is a $p$-regular element) then, by our hypotheses, there exists some $n \in N$ such that $\langle y\rangle^{n} \leq$ $\mathrm{C}_{G}(x)$, and so $x \in \mathrm{C}_{N}\left(\langle y\rangle^{n}\right)=\left(\mathrm{C}_{N}(\langle y\rangle)^{n}\right.$, a contradiction.

Lemma $22|N||\langle y\rangle||A \cap B|=\left|\frac{G}{N}\right||N \cap A||N \cap B|$.

Proof Recall that $p \in \pi(A N) \backslash \pi(B N)$ and $G=A N=B P N$ for any $P \in \operatorname{Syl}_{p}(G)$. Hence $G=A N=B\langle y\rangle N$. Now it is enough to make some computations having in mind that $|B \cap\langle y\rangle N|=|B \cap N|$ (recall that $G$ is $p$-separable).

Lemma 23 The Sylow p-subgroups of $G$ are cyclic of order $p$, i.e. $\langle y\rangle \cong C_{p}$.

Proof Take $x \in\langle y\rangle$ of order $p$, and set $H:=B N\langle x\rangle$; this is a subgroup of $G$ since $B N=$ $\mathrm{O}_{p^{\prime}}(G) \unlhd G$. Assume that $H=(H \cap A) B<G$. Now if $h \in(H \cap A) \cup B$ is $p$-regular of prime power order, by the hypotheses there exists $n \in N$ with $\langle x\rangle^{n} \leq\langle y\rangle^{n} \leq \mathrm{C}_{G}(h)$, so $h \in \mathrm{C}_{H}\left(\langle x\rangle^{n}\right)$, where $\langle x\rangle^{n} \in \operatorname{Syl}_{p}(H)$. By minimality, $\langle x\rangle=\mathrm{O}_{p}(H)$, so (recall that $G=B P N$, by Lemma 16 , with $P=\langle y\rangle) 1 \neq \mathrm{O}_{p}(H)^{G}=\mathrm{O}_{p}(H)^{B N P}=\mathrm{O}_{p}(H)^{P} \leq P$. This contradicts the fact $\mathrm{O}_{p}(G)=1$ and it proves the claim.

Lemma 24 The subgroup $\langle y\rangle$ does not normalise $N_{i}$, for each $i \in\{1, \ldots, r\}$. In particular, $r>1$.

Proof Assume that $\langle y\rangle$ normalises some $N_{i}$ with $i \in\{1, \ldots, r\}$. Then $\langle y\rangle$ normalises $N_{i}$ for each $i \in\{1, \ldots, r\}$. We can view $\langle y\rangle$ as a subgroup of $\operatorname{Aut}\left(N_{i}\right)$, because $\mathrm{C}_{\langle y\rangle}\left(N_{i}\right)=$ 1 (recall that $y$ has order $p$ ). By Lemma 9, there exists a prime $s \in \pi\left(N_{i}\right) \backslash \pi\left(\operatorname{Out}\left(N_{i}\right)\right)$ such that $\left(s,\left|\mathrm{C}_{N_{i}}(y)\right|\right)=1$. Therefore $s$ cannot divide $\left|\mathrm{C}_{N}(y)\right|$ as $\mathrm{C}_{N}(y)=\mathrm{C}_{N_{1}}(y) \times$ $\cdots \times \mathrm{C}_{N_{r}}(y)$.

Since each element of prime power order in $(N \cap A) \cup(N \cap B)$ centralises some Sylow $p$-subgroup (because our hypotheses), we deduce that $\pi(N \cap A) \cup \pi(N \cap B) \subseteq$ $\pi\left(\mathrm{C}_{N}(y)\right)$. Thus, this last property and Lemma 22 yield $s \in \pi(G / N)$.

Note that $G / N \lesssim \operatorname{Out}(N)$ and $\operatorname{Out}(N) \cong \operatorname{Out}\left(N_{1}\right)$ wr $\Sigma_{r}$, , the natural wreath product of $\operatorname{Out}\left(N_{1}\right)$ with $\Sigma_{r}$. As $s$ does not divide $\left|\operatorname{Out}\left(N_{i}\right)\right|$ for any $i$, it follows that 
$s \in \pi\left(\Sigma_{r}\right)$. Using Lemma 22, we obtain that $|N|_{s}$ divides $|G / N|_{s}$, and so it divides $\left|\Sigma_{r}\right|_{s}$. Set $\left|N_{1}\right|_{s}:=s^{d}$. Then $|N|_{s}=s^{d r}$ divides $s^{\frac{r-1}{s-1}}$, by Lemma 4 , so $d r \leq \frac{r-1}{s-1}$ and necessarily $d=0$, which contradicts the fact that $s$ divides $\left|N_{i}\right|$.

Lemma 25 Set $C:=\mathrm{C}_{\mathrm{O}_{p^{\prime}}(G) \cap A}(y)=\mathrm{C}_{\mathrm{O}_{p^{\prime}}(A)}(y)$ and $A_{0}:=\langle y\rangle \times C$. Then $A=(N \cap$ A) $A_{0}$ and $G=A N=A_{0} N$.

Proof By the minimal choice of $G$, we deduce that $G / N \cong A / A \cap N$ is $p$-decomposable. Hence $\left[\mathrm{O}_{p^{\prime}}(G) \cap A,\langle y\rangle\right] \leq N \cap A$. Thus, by coprime action, $\mathrm{O}_{p^{\prime}}(G) \cap A=\left[\mathrm{O}_{p^{\prime}}(G) \cap\right.$ $A,\langle y\rangle] \mathrm{C}_{\mathrm{O}_{p^{\prime}}(G) \cap A}(y) \leq(N \cap A) \mathrm{C}_{\mathrm{O}_{p^{\prime}}(G) \cap A}(y)$. Hence $A=(N \cap A) \mathrm{C}_{\mathrm{O}_{p^{\prime}}(G) \cap A}(y)\langle y\rangle=$ $(N \cap A) A_{0}$, and the assertion follows.

Recall that $N=N_{1} \times N_{2} \times \cdots \times N_{r}$ with $N_{i} \cong N_{1}$ a non-abelian simple group, and set $\Omega:=\left\{N_{1}, N_{2}, \ldots, N_{r}\right\}$. By Lemma $24, r>1$. As $G=A_{0} N, A_{0}$ acts transitively on $\Omega$. We adapt here some arguments used in [16] and we claim some facts about this action:

(i) The orbits of $B$ on $\Omega$ are the same as those of $C$.

This is clear because $\mathrm{O}_{p^{\prime}}(G)=\mathrm{O}_{p^{\prime}}(A) N=C N=B N$.

(ii) Let $\Delta$ be an orbit of $C$ on $\Omega$ of minimal lenght. If $c \in C$, then $\Delta^{y c}=\Delta^{c y}=\Delta^{y}$, so $\Delta^{y}$ and $\Delta \cap \Delta^{y}$ are also orbits of $C$. Therefore, by the choice of $\Delta$, either $\Delta=\Delta^{y}$ (and hence $\Delta=\Delta^{y^{i}}$ for $i \in\{1, \ldots, p\}$ ), or $\Delta \cap \Delta^{y}=\emptyset$ (and hence $\Delta^{y^{i}} \cap \Delta^{y^{j}}=\emptyset$ for $i \neq j, i, j \in\{1, \ldots, p\}$ ). It follows that there is a partition of $\Omega$ of the form

$$
\Omega=\Delta_{1} \cup \Delta_{2} \cup \cdots \cup \Delta_{k}
$$

where $\Delta_{i}:=\Delta^{y^{i-1}}$ for $i \in\{1, \ldots, k\}$, and $k \in\{1, p\}$. Note that all $\Delta_{i}$ have the same length, say $m$, and $\left\{\Delta_{1}, \ldots, \Delta_{k}\right\}$ are all the $C$-orbits (and $B$-orbits) on $\Omega$. Note also that $m$ is a $p^{\prime}$-number, since $C$ is a $p^{\prime}$-subgroup.

Moreover, $\langle y\rangle$ acts transitively on $\left\{\Delta_{1}, \Delta_{2}, \ldots, \Delta_{k}\right\}$.

(iii) The length of an orbit $\nabla$ of $\langle y\rangle$ on $\Omega$ is $k=p$, and there are $m$ orbits $\nabla_{1}:=\nabla$, $\nabla_{2} \ldots, \nabla_{m}$. Hence there is a partition

$$
\Omega=\nabla_{1} \cup \cdots \cup \nabla_{m}
$$

and both $\mathrm{O}_{p^{\prime}}(A)$ and $B$ act transitively on the set $\left\{\nabla_{1}, \nabla_{2}, \ldots, \nabla_{m}\right\}$. In particular, for each $1 \leq i \leq m$, there exists $a_{i} \in \mathrm{O}_{p^{\prime}}(A)$ such that $\nabla_{1}^{a_{i}}=\nabla_{i} ; a_{1}=1$.

Since the lenght of an orbit of $\langle y\rangle$ on $\Omega$ divides $p$ and $\langle y\rangle$ does not normalise any $N_{i}$, by Lemma 24, the first assertion follows. Now, the fact that $G=\langle y\rangle \mathrm{O}_{p^{\prime}}(A) N=$ $\langle y\rangle B N$ gives the last assertion.

(iv) It follows from (ii) and (iii) that $r=p m$, with $1=(m, p)$.

(v) Without loss of generality, we may consider $\Delta=\left\{N_{1}, \ldots, N_{m}\right\}$, and we set $M_{\Delta}:=$ $N_{1} \times \cdots \times N_{m}$. Then $M_{\Delta}$ is a minimal normal subgroup of $N C$.

Moreover, if $1 \neq R \leq N$ and $R \unlhd N C$, then there exist $\left\{x_{1}, \ldots, x_{d}\right\} \subseteq\langle y\rangle$ such that $R=M_{\Delta}^{x_{1}} \times \cdots \times M_{\Delta}^{x_{d}}$. 
(vi) Since $r>1$, then $m>1$.

Recall that $r>1$, by Lemma 24. If $m=1$, then $\langle y\rangle$ has only one orbit on $\Omega$, i.e. $\langle y\rangle$ acts transitively on $\Omega=\left\{N_{1}, \ldots, N_{k}\right\}$. Suppose, for instance, that $N_{i}=N_{1}^{y}$, for $i>1$. If there exists a non-trivial element $x \in \mathrm{C}_{N_{1}}(y)$, then $x=x^{y} \in N_{1} \cap N_{1}^{y}=$ $N_{1} \cap N_{i}$, a contradiction. Hence $\mathrm{C}_{N_{1}}(y)=1$, and it follows that $\mathrm{C}_{N}(y)=1$. But since $N \cap A \neq 1$, by Lemma 20, we can choose an element of prime powe order $1 \neq x \in N \cap A$ such that $x \in C_{N}(y)$ because the hypotheses on the indices (recall that $N$ is a $p^{\prime}$-group), which gives a contradiction.

(vii) Since $k=p>1$, then $A \cap M_{\Delta}=1=\mathrm{C}_{\mathrm{O}_{p^{\prime}}(A)}\left(M_{\Delta}\right)$.

Let $x \in A \cap M_{\Delta}$ of prime power order, which is a $p$-regular element. Then by hypotheses there exists $n \in N$ with $x \in \mathrm{C}_{G}\left(\langle y\rangle^{n}\right)$. Hence $x^{y^{n}}=x \in M_{\Delta} \cap M_{\Delta}^{y^{n}}=$ $M_{\Delta} \cap M_{\Delta}^{y}=1$. We deduce that $A \cap M_{\Delta}=1$.

Let $x \in \mathrm{C}_{\mathrm{O}_{p^{\prime}}(A)}\left(M_{\Delta}\right)$ of prime power order. Then there exists $n \in N$ such that $\left[\langle y\rangle^{n}, x\right]=1$. Therefore $\left[x, M_{\Delta}\right]=1=\left[x^{\left(y^{j}\right)^{n}}, M_{\Delta}^{\left(y^{j}\right)^{n}}\right]=\left[x, M_{\Delta}^{y^{j}}\right]$, for every $j \in$ $\{1, \ldots, p-1\}$. So $x \in \mathrm{C}_{G}(N)=1$. Hence $\mathrm{C}_{\mathrm{O}_{p^{\prime}}(A)}\left(M_{\Delta}\right)=1$.

(viii) Without loss of generality, let $\nabla=\left\{N_{1}, \ldots, N_{p}\right\}$. Set $M_{\nabla}:=N_{1} \times \cdots \times N_{p}$. Then $M_{\nabla}$ is a minimal normal subgroup of $N\langle y\rangle$, and if $1 \neq R \leq N$ with $R \unlhd N\langle y\rangle$, then there exist $\left\{d_{1}, \ldots, d_{t}\right\} \subseteq\left\{a_{1}, \ldots, a_{m}\right\} \subseteq \mathrm{O}_{p^{\prime}}(A)$ such that $R=M_{\nabla}^{d_{1}} \times \cdots \times M_{\nabla}^{d_{t}}$. Moreover, if we set $F_{1}:=N_{2} \times \cdots \times N_{p}, F_{i}:=F_{1}^{a_{i}}$ for each $2 \leq i \leq m$, and $F_{\nabla}:=$ $F_{1} \times \cdots \times F_{m}$, then $F_{\nabla} \cap \mathrm{O}_{p^{\prime}}(A)=1=F_{\nabla} \cap B$.

The first assertion is clear. If $x \in F_{\nabla} \cap \mathrm{O}_{p^{\prime}}(A)$ is of prime power order, then there exists $n \in N$ such that $\langle y\rangle^{n}$ centralises $x$, so for every $1 \leq j \leq p$ we get $x=x^{\left(y^{j}\right)^{n}} \in$ $F_{\nabla} \cap F_{\nabla}^{y^{j}} \leq E_{\nabla}:=\cap_{g \in\langle y\rangle} F_{\nabla}^{g}$. It follows that $F_{\nabla} \cap \mathrm{O}_{p^{\prime}}(A) \leq E_{\nabla}$. Note that $E_{\nabla} \leq N$ and it is normal in $N\langle y\rangle$, hence we deduce from the above that $E_{\nabla}=1$, and so $F_{\nabla} \cap \mathrm{O}_{p^{\prime}}(A)=1$. Analogously, $F_{\nabla} \cap B=1$.

Now, we will use the above facts on the actions of $C$ (and so $B$ ) and $\langle y\rangle$ on the set $\Omega$ to see that the minimal normal subgroup $N$ in our minimal counterexample cannot be a $p^{\prime}$-group.

The proof of the next Lemma follows similar arguments as those in [16, Lemma 11], using (i)-(viii) above, with suitable changes. However, we include an outline of the proof for the convenience of the reader.

Lemma 26 Let $s \neq p$ be a prime, and assume that $\left|N_{1}\right|_{s}=s^{n}$ and $\left|\operatorname{Out}\left(N_{1}\right)\right|_{s}=s^{\delta}$. Then $n(p-2) \leq \delta+\frac{m-1}{m(s-1)}$, where $r=$ pm. In particular, $n(p-2)<\delta+1$.

Proof Recall that a $\langle y\rangle$-orbit $\nabla$ on $\Omega$ has length $k=p>1$. Let $A_{s} \in \operatorname{Syl}_{s}(A)$ and $B_{s} \in \operatorname{Syl}_{s}(B)$. Note that $A_{s} \leq \mathrm{O}_{p^{\prime}}(A)$. By (viii) above, $F_{\nabla} \unlhd N$ and $F_{\nabla} \cap N \cap A_{s} \leq$ $F_{\nabla} \cap \mathrm{O}_{p^{\prime}}(A)=1$. So it follows that $\left|A_{s} \cap N\right| \leq\left|N: F_{\nabla}\right|_{s}=\left|N_{1}\right|_{s}^{m}=s^{n m}$. Analogously $\left|B_{S} \cap N\right| \leq s^{n m}$.

Set $M:=M_{\Delta}=N_{1} \times \cdots \times N_{m}$. From (v) and (vii) above we have that $M \unlhd N \mathrm{O}_{p^{\prime}}(A)=$ $N B$ and $M \cap \mathrm{O}_{p^{\prime}}(A)=1=\mathrm{C}_{\mathrm{O}_{p^{\prime}}(A)}(M)$. Hence $\mathrm{O}_{p^{\prime}}(A) \cong \mathrm{O}_{p^{\prime}}(A) \mathrm{C}_{G}(M) / \mathrm{C}_{G}(M) \lesssim$ $\operatorname{Aut}(M)$. Moreover $\operatorname{Aut}(M) \cong\left[\operatorname{Aut}\left(N_{1}\right) \times \cdots \times \operatorname{Aut}\left(N_{m}\right)\right] \Sigma_{m}$ $\cong \operatorname{Aut}\left(N_{1}\right)$ wr $\Sigma_{m}$, the natural wreath product of $\operatorname{Aut}\left(N_{1}\right)$ with $\Sigma_{m}$. Now applying Lemma 4 we deduce that $\left|A_{s}\right|$ divides $|\operatorname{Aut}(M)|$, and so $s^{(\delta+n) m} \cdot s^{\frac{m-1}{s-1}}$. 
On the other hand, if $|G / N|_{s}=s^{\gamma}$, then $|G|_{s}=|G / N|_{s}|N|_{s}=s^{\gamma+n r}$. Further, $\left|B_{s}\right|=|G / N|_{s}\left|B_{s} \cap N\right|$ divides $s^{\gamma+n m}$. Since $|G|_{s}$ divides $\left|A_{s}\right|\left|B_{s}\right|$, so $s^{\gamma+n r}$ divides $s^{\frac{m-1}{s-1}} \cdot s^{\gamma+n m} S^{(\delta+n) m}$. This fact, after some straightforward computations, leads to the desired conclusion, having in mind that $r=p m$.

Lemma $27 N$ is not a $p^{\prime}$-group.

Proof We take a prime $s \in \pi\left(N_{1}\right) \backslash \pi\left(\right.$ Out $\left.\left(N_{1}\right)\right)$ (such prime always exists, see for instance [16, Lemma 5]). Note that $s \neq p$, since $N$ is a $p^{\prime}$-group. Applying the previous Lemma for such prime we obtain that $n(p-2)<\delta+1$, but $\delta=0$ so necessarily $p=2$. This cannot happen, as it would imply that $N$ is a $2^{\prime}$-group, so soluble, which is a contradiction by Lemma 21 .

\section{The almost simple case}

Let $N$ be a non-abelian simple group with $p \in \pi(N)$, and let $N \unlhd G \leq \operatorname{Aut}(N)$ such that $G=A B=A N=B N$. Assume that $G$ satisfies the hypotheses of our main theorem, i.e. $p$ does not divide $i_{G}(x)$ for every $p$-regular element of prime power order $x \in A \cup B$.

We will carry out a case-by-case analysis of the simple group $N$ occuring as the socle of $G$ to prove that there is no a counterexample to our Main Theorem. Our strategy will apply the following lemma and the results in Section 3.

Lemma 28 For any $P \in \operatorname{Syl}_{p}(G)$

$$
\pi(G)=\pi\left(C_{G}(P)\right)=\pi(G / N) \cup \pi\left(C_{N}(P)\right) .
$$

In particular, $p \in \mathrm{Z}(\Gamma(G))$. Moreover, if $r \in \pi(G) \backslash \pi(G / N)$, then $r$ is adjacent to $p$ in $\Gamma(N)$.

Proof By our hypotheses, for any prime $r \in \pi(G) \backslash\{p\}$ there exists an element $x \in$ $A \cup B$ of order $r$ such that $x \in C_{G}(P)$, for some $P \in \operatorname{Syl}_{p}(G)$. Hence the first equality follows. Now, observe that $\pi(G)=\pi(G / N) \cup \pi(N)$ and, since $G=A N=B N=A B$, after some computations we also obtain

$$
|N||A \cap B|=\left|\frac{G}{N}\right||N \cap A||N \cap B|
$$

But again our hypotheses lead to $\pi((N \cap A) \cup(N \cap B)) \backslash\{p\} \subseteq \pi\left(C_{N}(P)\right)$. Also, since $p \in \pi(N), 1 \neq Z(P) \cap N \leq C_{N}(P)$. Hence the second equality also holds.

It is clear then that $p \in \mathrm{Z}(\Gamma(G))$. Assume now that $r \in \pi(G) \backslash \pi(G / N)$, and so $r \in \pi(N)$. By the second equality, we deduce that $r \in \pi\left(C_{N}(P)\right)$ and since $p \in \pi(N)$ the last assertion follows.

Lemma $29 N$ is not an alternating group $A_{n}$. 
Proof Let $N=A_{n}$ and assume first $n \neq 6$, so $G=N=A_{n}$ or $G=\Sigma_{n}$. Because our hypotheses, we may assume that $\Gamma(G)$ is connected. As in Lemma 10 , let $k \geq 2$ be the largest positive integer such that $\{n, n-1, \ldots, n-k+1\}$ are consecutive composite numbers, $r:=n-k$ the largest prime divisor of $n !$, and $t$ the largest prime with $t \leq k$. Since $p \in \mathrm{Z}(\Gamma(G))$, then $p \leq t$ by Lemma 10, and so $r>\frac{n}{2}>k \geq t \geq p$.

We claim that $r \notin \pi\left(\mathrm{C}_{G}(P)\right)$, for $P \in \operatorname{Syl}_{p}(G)$. Let suppose first that $p \neq 2$. Assume that there exists an element $x \in G$ of order $r$ such that $P \leq \mathrm{C}_{G}(x)$. Since $\mathrm{C}_{G}(x)$ is isomorphic to a subgroup of $C_{r} \times \Sigma_{n-r}$ and $p \neq r$, then $|P|$ divides $\left|\Sigma_{n-r}\right|$, and so $\left|\Sigma_{n}: \Sigma_{n-r}\right|=n(n-1) \cdots(n-r+1)$ should be a $p^{\prime}$-number. But this is a contradiction, since $p<r$.

If $p=k=2$, then, by Lemma 10 , it should be $G=\Sigma_{n}$, so the above reasonings work as well. Finally, if $p=2$ and $k \geq 3$, then $r \geq 5$ and we can argue as above to get a contradiction since $\left|\Sigma_{n}: \Sigma_{n-r}\right|=n(n-1) \cdots(n-r+1)$ is divisible by 4 .

If $n=6$, by Lemma 11, the only case to be considered is $G=\operatorname{Aut}(N)$, and since $\mathrm{Z}(\Gamma(G))=\{2\}$ it should be $p=2$. But a Sylow 2-subgroup of $G$ is self-centralising, so we get a contradiction.

Lemma $30 N$ is not an sporadic group.

Proof Assume that $N$ is an sporadic group. Since $p \in \mathrm{Z}(\Gamma(G))$, we may assume, by Lemma 12, that either $N=J_{2}$ or $N=M c L, G=\operatorname{Aut}(N)$, and $p=2$. Now Lemma 28 implies that 2 is adjacent in $N$ to any prime $r \neq 2$, but this is a contradiction since $N$ has a self-centralising Sylow $s$-subgroup (take $s=7$ for $N=J_{2}$ and $s=11$ for $N=M c L$; see [6]).

Lemma $31 N$ is not a simple group of Lie type.

Proof If $N$ is a simple group of Lie type of characteristic $t$, first notice that the prime $p$ such that $\pi(G)=\pi\left(C_{G}(P)\right)$ should be different from $t$, because it is well known that a Sylow $t$-subgroup is self-centralising in $G$. Moreover, since $|N||A \cap B|=$ $\left|\frac{G}{N}\right||N \cap A||N \cap B|$ and $|N|_{t}>|\operatorname{Out}(N)|_{t}$, we get that $t \in \pi((N \cap A) \cup(N \cap B)) \subseteq$ $\pi\left(C_{N}(P)\right)$. This means that $t$ should be adjacent to $p$ in $\Gamma(N)$.

Now, we derive from Lemmas 13 and 14 that, apart from some exceptional cases that we consider below, either there exist a Sylow $s$-subgroup of $N$ of order $s \notin$ $\pi(\operatorname{Out}(N))$ which is self-centralising in $N$, or there exist two primes $r, s \in \pi(N) \backslash$ $\pi(G / N)$, and two maximal tori $T_{1}$ and $T_{2}$ of $N$ such that $r \in \pi\left(T_{1}\right), s \in \pi\left(T_{2}\right)$, and $\left(\left|T_{1}\right|,\left|T_{2}\right|\right)=1$. But from Lemma 28, $p$ is a prime which is adjacent both to $r$ and $s$ in $\Gamma(N)$, and therefore $p \in \pi\left(T_{1}\right) \cap \pi\left(T_{2}\right)$, which gives a contradiction.

For $N=L_{2}(q), q=t^{e}$, the fact that $C_{N}(x)$ is a $t$-group for any $t$-element $x \in N$, implies that $t$ is not adjacent in $\Gamma(N)$ to any other prime in $\pi(N)$, a contradiction.

If $N=L_{3}(q)$ or $N=U_{3}(q), q=t^{e}$, the assertion in Lemma 13 on the corresponding maximal torus $T$ in each case guarantees that $p \in \pi(T)$ is a primitive prime divisor of $q^{3}-1$ (respectively $q^{6}-1$ ) and $p$ is not adjacent to the prime $t$ in $\Gamma(N)$. In fact, $p$ is not adjacent in $\Gamma(N)$ to any prime $s \notin \pi(T)$, which gives a contradiction.

The Main Theorem is proved. 


\section{References}

1. Amberg B., Franciosi S., de Giovanni F.: Products of groups. Oxford University Press Inc., New York (1992)

2. Ballester-Bolinches A., Cossey J., Li Y.: Mutually permutable products and conjugacy classes, Monatsh. Math., 170, 305-310 (2013)

3. Ballester-Bolinches A., Esteban-Romero R., Asaad M.: Products of finite groups. Vol. 53 of de Gruyter Expositions in Mathematics, Berlin (2010)

4. Camina A.R., Camina R.D.: The influence of conjugacy class sizes on the structure of finite groups: a survey, Asian-Eur. J. Math., 4, 559-588 (2011)

5. Carter R.W.: Centralizers of semisimple elements in the finite classical groups, Proc. London Math. Soc., 42, 1-41 (1981)

6. Conway J.H., Curtis R.T., Norton S.P., Parker R.A., Wilson R.A.: Atlas of finite groups. Clarendon Press (1985)

7. Cossey J., Li Y.: On the structure of a mutually permutable product of finite groups, Acta Math Hungar., 154, 525-529 (2018)

8. Doerk K., Hawkes T.: Finite Soluble Groups. Vol. 4 of de Gruyter Expositions in Mathematics, Berlin (1992)

9. Dolfi S., Pacifici E., Sanus L., Spiga P.: On the orders of zeros of irreducible characters, J. Algebra, 321, 345-352 (2009).

10. Fein B., Kantor W.M., Schacher M.: Relative Brauer groups II, J. Reine Angew. Math., 328, 39-57 (1981)

11. Felipe M.J., Martínez-Pastor A., Ortiz-Sotomayor V.M.: Square-free class sizes in products of groups, J. Algebra, 491, 190-206 (2017)

12. Felipe M.J., Martínez-Pastor A., Ortiz-Sotomayor V.M.: Prime power indices in factorised groups, Mediterr. J. Math., 14, article: 225 (2017)

13. Felipe M.J., Martínez-Pastor A., Ortiz-Sotomayor V.M.: Zeros of irreducible characters in factorised groups, Ann. Mat. Pura Appl., 198, 129-142 (2019)

14. Felipe M.J., Martínez-Pastor A., Ortiz-Sotomayor V.M.: Products of groups and class sizes of $\pi$ elements, Mediterr. J. Math., 17, article: 15 (2020)

15. He H., Shi W.: A Note on the Adjacency Criterion for the Prime Graph and the Characterization of $C_{p}(3)$, Algebra Colloq., 19 (3), 553-562 (2012)

16. Kazarin L.S., Martínez-Pastor A., Pérez-Ramos M.D.: A reduction theorem for a conjecture on products of two $\pi$-decomposable groups, J. Algebra, 379, 301-313 (2013)

17. Kondratiev A.S.: On prime graph components of finite simple groups, Math. Sb., 67 (1), 235-247 (1990) Translation from Mat. Sb., 180 (6), 787-797 (1989)

18. Liebeck M., Praeger C.E., Saxl J.: The maximal factorizations of the finite simple groups and their automorphism groups, Mem. Amer. Math. Soc., 86 (432), Amer. Math. Soc., Providence, RI (1990)

19. Liu X., Wang Y., Wei H.: Notes on the length of conjugacy classes of finite groups, J. Pure Appl. Algebra, 196, 111-117 (2005)

20. Lucido M.S.: Prime graph components of finite almost simple groups, Rend. Semin. Mat. Univ. Padova, 102, 1-22 (1999) Addendum in: Rend. Semin. Mat. Univ. Padova, 107, 1-2 (2002)

21. Vasiliev A.V., Vdovin E.P.: An adjacency criterion for the prime graph of a finite simple group, Algebra and Logic, 44 (6), 381-406 (2005)

22. Vasiliev A.V., Vdovin E.P.: Cocliques of maximal size in the prime graph of a finite simple group, Algebra and Logic, 50 (4), 291-322 (2011)

23. Williams J.S.: Prime graph components of finite groups, J. Algebra, 69, 487-513 (1981)

24. Zavarnitsin A.V.: Recognition of the simple groups $L_{3}(q)$ by element orders, J. Group Theory, 7 , 81-97 (2004)

25. Zhao X.H., Guo X.Y., Shi J.Y.: On the conjugacy class sizes of prime power order $\pi$-elements, South. Asian Bull. Math., 35, 735-740 (2011)

26. Zsigmondy K.: Zur Theorie der Potenzreste, Monatsh. Math. Phys., 3, 265-284 (1892) 